\title{
Specific Bioactive Collagen Peptides in Osteopenia and Osteoporosis: Long-Term Observation in Postmenopausal Women
}

\author{
Denise Zdzieblik' ${ }^{1}$, Steffen Oesser ${ }^{1}$, Daniel König ${ }^{2,3}$ \\ ${ }^{1} \mathrm{CRI}$, Collagen Research Institute, Kiel, Germany \\ ${ }^{2}$ Center of Sports Science, Department for Nutrition, Exercise and Health, University of Vienna, Vienna; \\ ${ }^{3}$ Faculty of Life Sciences, Department for Nutrition, Exercise and Health, University of Vienna, Vienna, Austria
}

\section{Corresponding author \\ Denise Zdzieblik \\ CRI, Collagen Research Institute, \\ Schauenburgerstrasse 116, Kiel 24118, \\ Germany \\ Tel: +49-431-5606616 \\ Fax: +49-431-5606613 \\ E-mail: denise.zdzieblik@cri-mail.org}

Received: April 27, 2021

Revised: June 28, 2021

Accepted: June 30, 2021
Background: The effects of specific collagen peptides on bone mineral density (BMD) in subjects with osteoporosis or osteopenia have already been investigated in 131 postmenopausal women in a randomized controlled trial. The purpose of this follow-up observation was to determine the longer-term effects of the same specific bioactive collagen peptides after a total intervention time of 4 years. Methods: In this open-label follow-up observation, 31 postmenopausal women with reduced BMD (initial T-score lower than-1 of either the femoral neck or the lumbar spine) completed the follow-up. BMD was measured via dual energy X-ray absorptiometry. Absolute changes in BMD and Tscores in the spine and femoral neck were assessed. The number of fractures was also recorded. All participants received specific bioactive collagen peptides. Results: Supplementation with bioactive collagen peptides during follow-up led to a clinically relevant increase in BMD in the spine. These findings were consistent with the results for the femoral neck. Conclusions: Long-term supplementation with specific bioactive collagen peptides appears to be effective in counteracting losses in BMD. Moreover, significant increases in BMD could contribute to improved bone stability.

Key Words: Bone density · Collagen · DXA · Osteoporosis · Peptides

\section{INTRODUCTION}

Osteoporosis is characterized by an imbalance of anabolic and catabolic processes in the bone. The consequence is reduced mineralization of the bone and finally loss in bone stability. An increased osteoclast activity (high turnover), a reduced osteoblast activity (low turnover) individually or in combination (bone atrophy) can lead to an imbalance in bone metabolism.[1] The consequence of osteoporosis is an increased risk of fractures. Approximately $34 \%$ of all women - predominantly post-menopausal - suffer from osteoporosis.[2] Lack of exercise, malnutrition (especially reduced calcium and vitamin D supply), endocrinological disorders or the intake of medications can have a negative effect on the mineral balance of the bones.[3]

Physical activity [4,5] and an adequate protein intake ( $1 \mathrm{~g} / \mathrm{kg}$ body weight daily) [6] are basically recommended for osteoporosis prevention. In addition, a sufficient

\section{Mineral Research}

This is an Open Access article distributed under the terms of the Creative Commons Attribution Non-Commercial License (https://creativecommons.org/licenses/by-nc/4.0/ which permits unrestricted non-commercial use, distribution, and reproduction in any medium, provided the origina work is properly cited. 
intake of calcium ( $>1,200 \mathrm{mg} /$ day), vitamin D (800 lU) and vitamin $B 12$ should be taken into consideration. $[7,8]$ The basic therapy for osteoporosis is often inadequate since age-related bone loss results from hormonal changes. Hormone therapies, estrogen receptor modulators or bisphosphonates are, therefore, available for treatment. However, some of these drugs are often poorly tolerated and have serious side effects in individual cases.[7] Only $12.9 \%$ to $72.0 \%$ of all osteoporosis patients worldwide use treatment options within the first 2 years.[9] Therefore, well-tolerated and effective therapeutic approaches are of high importance.

For some years, dietary supplements and collagen peptides have gained increasing attention as alternative therapeutic measures in osteoporosis. The results of clinical studies have shown that the additional administration of collagen peptides increases the osteoprotective effect of calcitonin administration.[10] A recent study also demonstrated that bone mineral density (BMD) is improved by the simultaneous intake of collagen peptides, calcium, and vita$\min$ D.[11] Collagen peptides are thought to improve calcium retention $[12,13]$ and directly influence bone metabolism by releasing growth hormones and regulating the osteoblast function.[14]

König et al. [15] previously reported an osteoprotective effect of specific collagen peptides in 131 postmenopausal women with reduced BMD in the spine and femoral neck. After 1 year, the daily intake of $5 \mathrm{~g}$ of collagen peptides resulted in a statistically significant increase in the T-score considering the diagnosis of osteoporosis and the assessment of fracture risk. Changes in bone turnover markers indicated favourable anabolic processes in the bone. In contrast, in the placebo group, T-score and bone turnover markers showed a continuous decrease in BMD.[15]

The purpose of this follow-up observation was to determine the osteoprotective effect of $5 \mathrm{~g}$ specific collagen peptides (FORTIBONE ${ }^{\circledR}$; GELITA AG, Eberbach, Germany) in continuation of the study by König et al. [15].

\section{METHODS}

\section{Design and participants}

This investigation was a non-controlled, open-label follow-up observation. At the beginning of the follow-up, all participants received the specific bioactive collagen pep- tides.

Data from 31 postmenopausal (amenorrhea for at least 1 year) women with reduced BMD (initial dual energy X-ray absorptiometry [DXA] T-score of -1 or lower on either the femoral neck or the lumbar spine) were included in the statistical analysis.[15] Women with severe chronic disease, comorbidity or medically or endocrinologically induced osteoporosis were already excluded in the initial study. Reported medical treatment for osteoporosis within the last year was also defined as an exclusion criterion. In addition, unstable weight and eating behavior led to the exclusion of the examined subjects. Participation was also not possible if participants were allergic to the investigational product.

\section{Efficacy outcomes}

The changes in T-scores and BMD - determined as bone mineral content per area in $\mathrm{g} / \mathrm{cm}^{2}$ - in the spine (lumbar vertebrae 1-4) and the femoral neck were the outcome variables of the follow-up. For this purpose, differences between the start of the follow-up and the fourth year of intervention were compared. The T-scores and BMD were measured at the start and again after the second, third and fourth year of the follow-up using DXA (Stratos DR Dual Fan Beam, Degen Medizintechnik, Heppenheim, Germany).

Although DXA is the gold standard for determining BMD, the clinical relevance of osteoporosis is based on the development of fractures.[16] Therefore, the occurrence of fragility fractures was assessed, too.

At each study visit patients' self-reported adverse events were collected for safety analysis. The monitoring of the data entry was performed by the study centre. Any concerns were clarified directly with the study physician and the participants' medical reports.

\section{Investigational product}

The same bioactive collagen peptides (FORTIBONE ${ }^{\circledR}$ ) from the main randomised control trial with a high safety (GRAS status) were used for this intervention. The peptides derive from specific hydrolysis of type I collagen with a mean molecular weight of about $5 \mathrm{kDa}$ and are clearly defined by the molecular weight fraction and the amino acid profile. The test product was packed in single sachets containing a daily dose of $5 \mathrm{~g}$. The powders had to be dissolved in 250 $\mathrm{mL}$ of water at room temperature and ingested once daily. 


\section{Statistical analysis}

All data are presented as mean \pm standard deviation (SD) in tables. IBM SPSS Statistics (version 25.0; IBM Corp., Armonk, NY, USA) was used for all statistical analyses. All tests in the descriptive analysis were performed as 2-sided tests, and the significance level was set at $a=0.05$.

The changes in BMD and T-scores for spine and femur during the intervention period within the group were analyzed by repeated-measures ANOVA. The factor was time (start, first, second and third year of follow-up).

\section{RESULTS}

\section{Subjects}

Of the 131 subjects who completed the initial randomized controlled trial, 13 women of the former treatment group (group 1) and 18 women of the former placebo group starting the oral treatment with specific collagen peptides at the beginning of the follow-up (group 2) agreed to continue the follow-up. In total, 23 had completed the followup. Eight women of group 2 voluntarily withdrew from further participation. The drop-outs are listed in Figure 1. None of the drop-outs was related to any side effects or adverse events caused by taking the collagen peptide supplement. No serious adverse events were observed in either group. No adverse events were reported and, in particular, no pathological findings were observed in routine testing. Therefore, the investigational product appears safe and well-tolerated.

The data of the participants at the start of the follow-up are summarized in Table 1. No statistically significant differences for any parameter were observed between the 2 study groups at the beginning of the follow-up (Table 1).

\section{Changes in BMD}

As shown in Figure 2, the current investigation identified

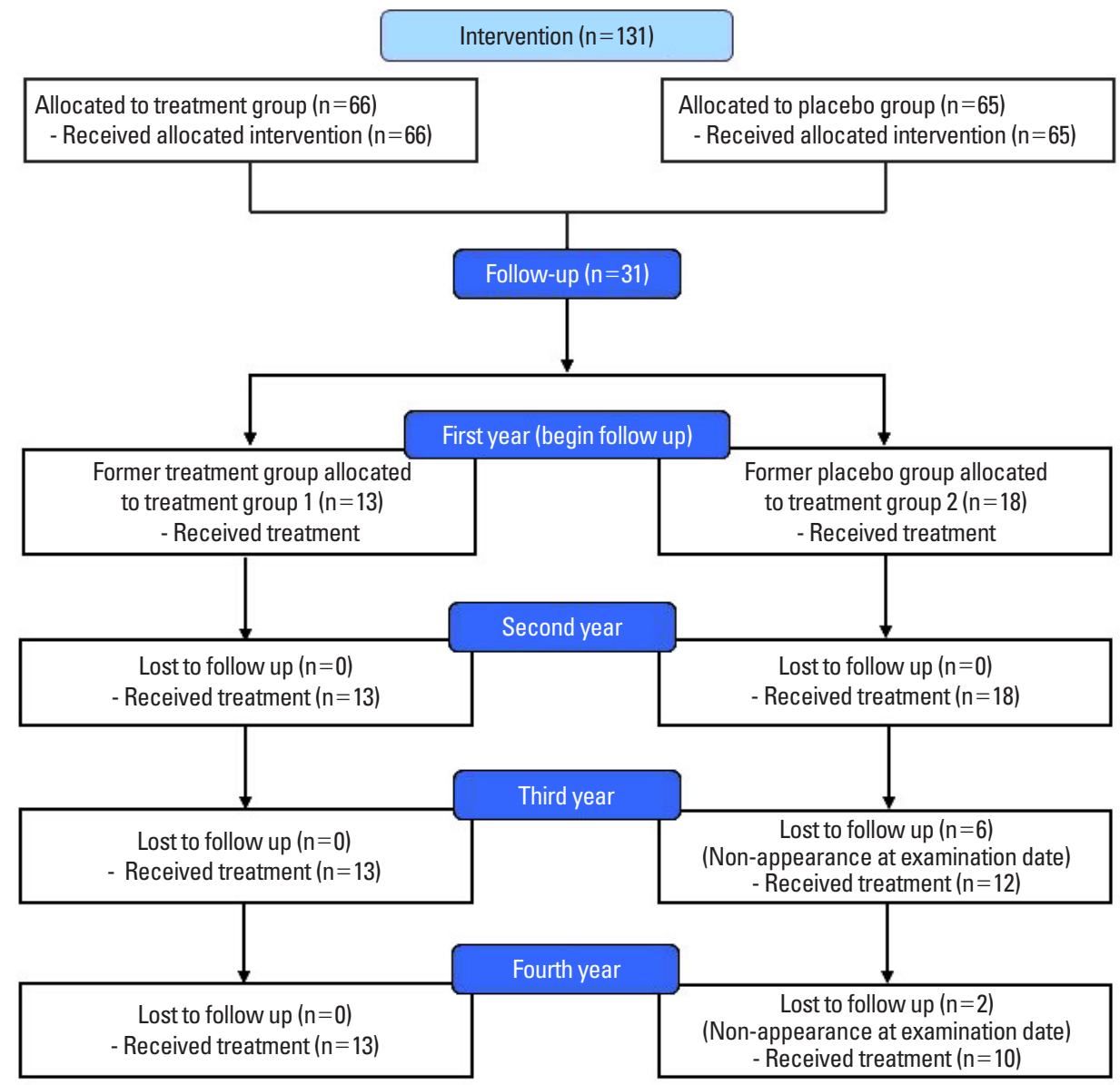

Fig. 1. Flow chart of participants (trial profiles). 
Table 1. Initial data for the follow-up population ( $N=31)$

\begin{tabular}{lcccc}
\hline & Total $(\mathrm{N}=31)$ & Group 1 $(\mathrm{N}=13)$ & Group 2 $(\mathrm{N}=18)$ & $P$-value \\
\hline Age $(\mathrm{yr})$ & $62.9 \pm 7.21$ & $62.9 \pm 8.57$ & $62.9 \pm 6.31$ & 0.987 \\
Height $(\mathrm{m})$ & $1.62 \pm 0.067$ & $1.62 \pm 0.061$ & $1.61 \pm 0.072$ & 0.505 \\
Body weight $(\mathrm{kg})$ & $61.4 \pm 9.42$ & $63.2 \pm 9.93$ & $60.2 \pm 9.11$ & 0.387 \\
BMI $\left(\mathrm{kg} / \mathrm{m}^{2}\right)$ & $23.6 \pm 4.10$ & $23.8 \pm 3.51$ & $23.4 \pm 4.57$ & 0.773 \\
RRsyst $(\mathrm{mmHg})$ & $123.4 \pm 27.5$ & $115.5 \pm 30.6$ & $128.6 \pm 24.7$ & 0.208 \\
RRdia $(\mathrm{mmHg})$ & $82.7 \pm 11.0$ & $79.2 \pm 9.00$ & $85.0 \pm 11.8$ & 0.157 \\
BMD spine $\left(\mathrm{g} \cdot \mathrm{cm}^{-2}\right)$ & $0.778 \pm 0.095$ & $0.773 \pm 0.108$ & $0.783 \pm 0.086$ & 0.784 \\
BMD femur $\left(\mathrm{g} \cdot \mathrm{cm}^{-2}\right)$ & $0.796 \pm 0.085$ & $0.815 \pm 0.101$ & $0.782 \pm 0.072$ & 0.307 \\
T-score spine & $-2.47 \pm 0.823$ & $-2.52 \pm 0.943$ & $-2.44 \pm 0.751$ & 0.784 \\
T-score femur & $-1.43 \pm 0.689$ & $-1.28 \pm 0.813$ & $-1.54 \pm 0.583$ & 0.304 \\
\hline
\end{tabular}

The data is presented as mean \pm standard deviation.

${ }^{a}$ Differences between groups tested with unpaired Student's $t$-test.

BMI, body mass index; BMD, bone mineral density; RRsyst, systolic blood pressure; RRdia, diastolic blood pressure.

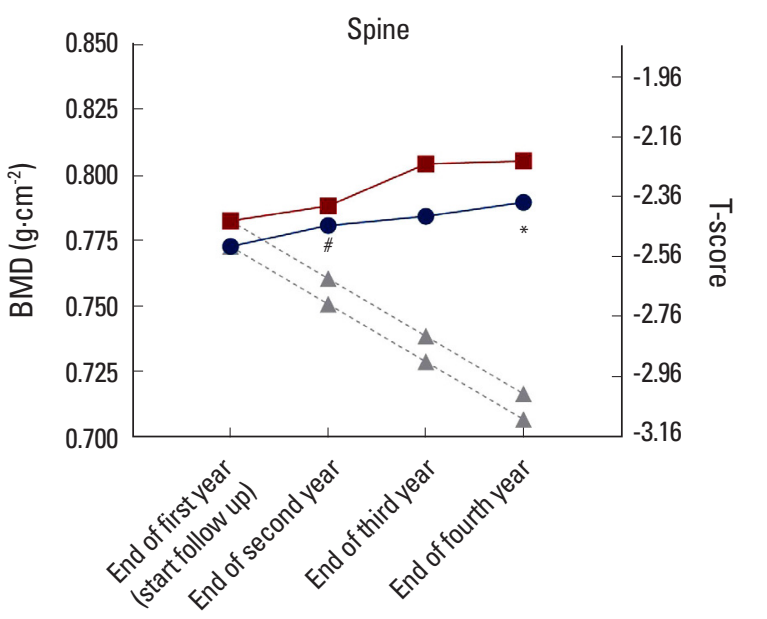

$$
\begin{aligned}
& \text { - Group } 1 \\
& - \text { Group } 2 \\
& \text { - Annual loss of normal population }
\end{aligned}
$$

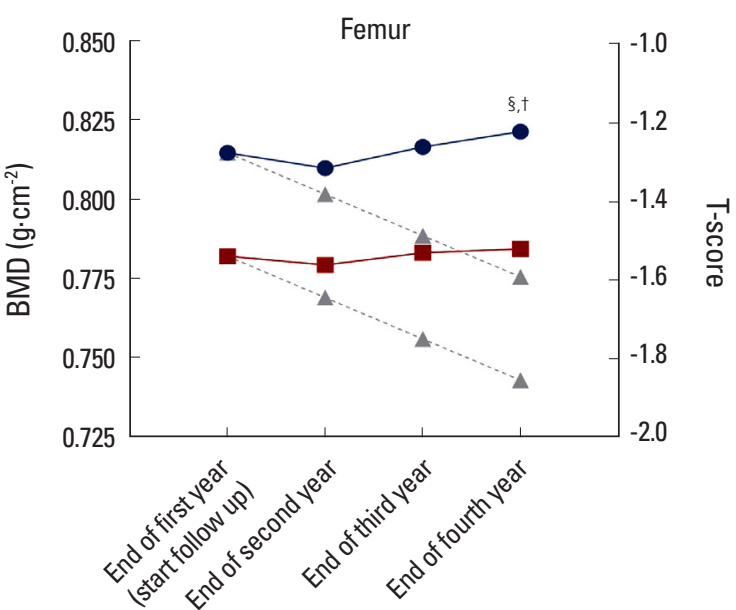

- Group 1

- Group 2

- Annual loss of normal population

Fig. 2. Changes in bone mineral density and T-scores for spine and femur from start of follow-up to 4th year of treatment. Data shown as mean. ${ }^{\#} P<0.05$ within group from start of follow-up to 2 nd year of treatment. ${ }^{*} P<0.05$ within group from start of follow-up to 4 th year of treatment. ${ }^{\S} P<0.05$ within group from 2 nd to 4 th year of treatment. ${ }^{\dagger} P<0.05$ within group from 3 rd to 4 th year of treatment. The annual loss of normal population without treatment according to Finkelstein et al. [18]. 
a clear improvement in the BMD of the spine and femur in both groups. From the start of follow-up to the second year $(P=0.006)$ and to the fourth year $(P=0.049)$ of treatment, changes in the BMD of the spine were statistically significant in group 1 (Fig. 2). Furthermore, changes in the $B M D$ of the femoral neck reached the level of statistical significance within group 1 from the second to the fourth year of treatment $(P=0.040)$ and from the third to the fourth year of treatment $(P=0.018)$. In group 2 tendency towards significance was demonstrated for changes in the BMD of the femoral neck from the second to the fourth year of treatment $(P=0.052)$. These results are in line with the changes in the T-scores of the respective measurement sites (Fig. 2).

The relative changes in spine and femoral neck during the total follow-up period was $5.79 \%$ and $4.21 \%$ for group 1 , respectively. In group 2, the results of the DXA revealed an improvement of $8.16 \%$ in the spine and $1.23 \%$ in the femoral neck. In group 1 the relative changes in the spine showed a tendency towards significance $(P=0.059)$. For all other measurements, the relative changes did not reach the level of statistical significance.

None of the participants within either group 1 or 2 had osteoporotic fractures during the follow-up period.

\section{DISCUSSION}

In the follow-up, a daily intake of $5 \mathrm{~g}$ of specific collagen peptides showed a progressive increase in BMD, indicating an increased anabolic bone metabolism in postmenopausal women after a total of 4 years. The results are consistent with the findings of the study by König et al. [15], on which this observational follow-up was based.

As the magnitude of the change in the BMD, the least significant change has to be considered. Since the DXA measurement has an estimated precision of $1.1 \%$ in the respective measurement sites, a clinically relevant change in BMD has to be at least 3\%.[17] In the current follow-up observation, BMD increased by $5.79 \%$ to $8.16 \%$ in the spine and by $1.23 \%$ to $4.21 \%$ in the femoral neck. Moreover, it is known from the literature that in postmenopausal women the average annual loss of BMD in the spine and hip is 0.022 and $0.013 \mathrm{~g} / \mathrm{cm}^{2}$.[18] Taking into account these changes in the postmenopause, the improvement in BMD in the spine and femoral neck was $7 \%$ in relation to the loss of BMD known from the literature indicating a pronounced, clini- cally relevant effect.[19] The positive impact of the specific collagen peptides on BMD and its clinical relevance in osteoporosis treatment was supported by the absence of any fractures during the follow-up period.

To date, there is only a limited number of clinical studies that investigate the osteoprotective effect of collagen peptides. Previous clinical studies have only shown a synergistic effect of collagen peptides with calcium, vitamin $D$ and calcitonin therapy.[10,11] Preclinical studies have shown that the single administration of collagen peptides has a beneficial effect on bone metabolism.[20,21]

According to the current state of research, the positive effect of collagen peptides in cartilage,[22-26] tendon and ligament [27] tissue may be due to molecular biological processes such as the stimulation of elastin, collagen type I and III formation.

Collagen structures also play an important role in bone development. They bind and store growth factors and cytokines. For example, insulin-like growth factor-I and II are linked by collagen in the bone. Collagen networks are broken down during bone remodeling. As a result, growth factors, which in turn promote bone formation, are released. The Asp-Gly-Glu-Ala peptides, which are also derived from collagen, are able to trigger the formation of osteoblasts from bone marrow cells. Hyp-peptides also promote osteoblast activity, resulting in increased bone mineralization and the synthesis of organic bone components.[28] Future research is warranted to examine the influence of collagen peptides in bone metabolism at the cellular level.

This follow-up observation has some limitations. The small sample size reduces the power and increases the margin of error. Since various collagen peptide products differ in their composition and hence their bioavailability and mode of action, the respective effects of the follow-up observation cannot be assumed for all collagen peptides.

\section{CONCLUSIONS}

The daily intake of $5 \mathrm{~g}$ of specific bioactive collagen peptides resulted in a steady increase in BMD and the T-score in the spine and femoral neck after 4 years. Previously known therapy options are sometimes poorly tolerated and, in some cases, associated with severe side effects. Although further research is needed, specific bioactive collagen peptides (FORTIBONE ${ }^{\circledR}$ ) could offer an innovative therapeutic 
approach for a long-term osteoporosis treatment with good tolerability without the potential side effects of some forms of drug treatment.

\section{DECLARATIONS}

\section{Acknowledgments}

We would like to thank all the participants as well as the staff of the University of Freiburg who supported us with the examination.

\section{Funding}

The specific collagen peptides (product name FORTIBONE $^{\oplus}$ ) were provided by the GELITA GmbH, Eberbach Germany.

\section{Ethics approval and consent to participate}

The original interventional study (DRKS00009708) was conducted according to the guidelines of the Declaration of Helsinki, and approved by the independent Ethics Committee of the University of Freiburg.

\section{Authors' contributions}

Conceptualization: DZ, SO, and DK; Data curation: DZ and DK; Formal analysis: DZ and DK; Writing-original draft preparation: DZ, SO, and DK; Writing-review and editing: DZ, SO, and DK; All authors read and approved the final manuscript.

\section{Conflict of interest}

No potential conflict of interest relevant to this article was reported. SO has given scientific lectures that were in parts supported by the GELITA AG. He is also co-inventor of patents concerning the use of collagen peptides. All procedures performed in studies involving human participants were in accordance with the ethical standards of the institutional and/or national research committee, and with the 1964 Helsinki declaration and its later amendments or comparable ethical standards. The funders had no role in the design of the study; in the collection, analyses, or interpretation of data; in the writing of the manuscript, or in the decision to publish the results. The authors responsible for data assessment and analysis have neither financial nor competing interests concerning the outcome of this investigation.

\section{ORCID}

Denise Zdzieblik https://orcid.org/0000-0001-8637-8757

Daniel König https://orcid.org/0000-0001-7002-1286

\section{REFERENCES}

1. Boskey AL, DiCarlo E, Paschalis E, et al. Comparison of mineral quality and quantity in iliac crest biopsies from highand low-turnover osteoporosis: an FT-IR microspectroscopic investigation. Osteoporos Int 2005;16:2031-8. https:// doi.org/10.1007/s00198-005-1992-3.

2. Johnell O, Kanis JA. An estimate of the worldwide prevalence and disability associated with osteoporotic fractures. Osteoporos Int 2006;17:1726-33. https://doi.org/10.1007/ s00198-006-0172-4.

3. World Health Organization. Prevention and management of osteoporosis. World Health Organ Tech Rep Ser 2003; 921:1-164, back cover.

4. Guadalupe-Grau A, Fuentes T, Guerra B, et al. Exercise and bone mass in adults. Sports Med 2009;39:439-68. https:// doi.org/10.2165/00007256-200939060-00002.

5. Todd JA, Robinson RJ. Osteoporosis and exercise. Postgrad Med J 2003;79:320-3. https://doi.org/10.1136/pmj.79.932. 320.

6. Mangano KM, Sahni S, Kerstetter JE. Dietary protein is beneficial to bone health under conditions of adequate calcium intake: an update on clinical research. Curr Opin Clin Nutr Metab Care 2014;17:69-74. https://doi.org/10.1097/ mco.0000000000000013.

7. Tella SH, Gallagher JC. Prevention and treatment of postmenopausal osteoporosis. J Steroid Biochem Mol Biol 2014; 142:155-70. https://doi.org/10.1016/j.jsbmb.2013.09.008.

8. Hamrick I, Schrager S, Nye AM. Treatment of osteoporosis: current state of the art. Wien Med Wochenschr 2015;165: 54-64. https://doi.org/10.1007/s10354-014-0335-4.

9. Fatoye F, Smith P, Gebrye T, et al. Real-world persistence and adherence with oral bisphosphonates for osteoporosis: a systematic review. BMJ Open 2019;9:e027049. https: //doi.org/10.1136/bmjopen-2018-027049.

10. Adam M, Spacek P, Hulejová H, et al. Postmenopausal osteoporosis. Treatment with calcitonin and a diet rich in collagen proteins. Cas Lek Cesk 1996;135:74-8.

11. Elam ML, Johnson SA, Hooshmand S, et al. A calcium-collagen chelate dietary supplement attenuates bone loss in postmenopausal women with osteopenia: a randomized 
controlled trial. J Med Food 2015;18:324-31. https://doi. org/10.1089/jmf.2014.0100.

12. Harnedy PA, FitzGerald RJ. Bioactive peptides from marine processing waste and shellfish: A review. J Funct Foods 2012;4:6-24. https://doi.org/10.1016/j.jff.2011.09.001.

13. Kim SK, Mendis E. Bioactive compounds from marine processing byproducts: A review. Food Res Int 2006;39:38393. https://doi.org/10.1016/j.foodres.2005.10.010.

14. Mizuno M, Kuboki Y. Osteoblast-related gene expression of bone marrow cells during the osteoblastic differentiation induced by type I collagen. J Biochem 2001;129:1338. https://doi.org/10.1093/oxfordjournals.jbchem.a002824.

15. König D, Oesser S, Scharla S, et al. Specific collagen peptides improve bone mineral density and bone markers in postmenopausal women: A randomized controlled study. Nutrients 2018;10. https://doi.org/10.3390/nu10010097.

16. Kanis JA, McCloskey EV, Johansson $\mathrm{H}$, et al. European guidance for the diagnosis and management of osteoporosis in postmenopausal women. Osteoporos Int 2013;24:2357. https://doi.org/10.1007/s00198-012-2074-y.

17. Baim S, Wilson CR, Lewiecki EM, et al. Precision assessment and radiation safety for dual-energy $\mathrm{X}$-ray absorptiometry: position paper of the International Society for Clinical Densitometry. J Clin Densitom 2005;8:371-8. https://doi. org/10.1385/jcd:8:4:371.

18. Finkelstein JS, Brockwell SE, Mehta V, et al. Bone mineral density changes during the menopause transition in a multiethnic cohort of women. J Clin Endocrinol Metab 2008;93: 861-8. https://doi.org/10.1210/jc.2007-1876.

19. Liberman UA, Weiss SR, Bröll J, et al. Effect of oral alendronate on bone mineral density and the incidence of fractures in postmenopausal osteoporosis. The Alendronate Phase III Osteoporosis Treatment Study Group. N Engl J Med 1995;333:1437-43. https://doi.org/10.1056/nejm1995 11303332201.

20. Kim HK, Kim MG, Leem KH. Osteogenic activity of collagen peptide via ERK/MAPK pathway mediated boosting of collagen synthesis and its therapeutic efficacy in osteoporotic bone by back-scattered electron imaging and mi- croarchitecture analysis. Molecules 2013;18:15474-89. https://doi.org/10.3390/molecules181215474.

21. Watanabe-Kamiyama M, Shimizu M, Kamiyama S, et al. Absorption and effectiveness of orally administered low molecular weight collagen hydrolysate in rats. J Agric Food Chem 2010;58:835-41. https://doi.org/10.1021/jf9031487.

22. Nakatani S, Mano H, Sampei C, et al. Chondroprotective effect of the bioactive peptide prolyl-hydroxyproline in mouse articular cartilage in vitro and in vivo. Osteoarthritis Cartilage 2009;17:1620-7. https://doi.org/10.1016/j.joca. 2009.07.001.

23. Ng KW, Saliman JD, Lin EY, et al. Culture duration modulates collagen hydrolysate-induced tissue remodeling in chondrocyte-seeded agarose hydrogels. Ann Biomed Eng 2007;35:1914-23. https://doi.org/10.1007/s10439-0079373-z.

24. Oesser S, Seifert J. Stimulation of type II collagen biosynthesis and secretion in bovine chondrocytes cultured with degraded collagen. Cell Tissue Res 2003;311:393-9. https:// doi.org/10.1007/s00441-003-0702-8.

25. Ohara $\mathrm{H}$, Ichikawa $\mathrm{S}$, Matsumoto $\mathrm{H}$, et al. Collagen-derived dipeptide, proline-hydroxyproline, stimulates cell proliferation and hyaluronic acid synthesis in cultured human dermal fibroblasts. J Dermatol 2010;37:330-8. https://doi.org/ 10.1111/j.1346-8138.2010.00827.x.

26. McAlindon TE, Nuite $M$, Krishnan $N$, et al. Change in knee osteoarthritis cartilage detected by delayed gadolinium enhanced magnetic resonance imaging following treatment with collagen hydrolysate: a pilot randomized controlled trial. Osteoarthritis Cartilage 2011;19:399-405. https: //doi.org/10.1016/j.joca.2011.01.001.

27. Schunck M, Oesser S. Specific collagen peptides benefit the biosynthesis of matrix molecules of tendons and ligaments. J Int Soc Sports Nutr 2013;10:P23. https://doi.org/ 10.1186/1550-2783-10-S1-P23.

28. Daneault A, Prawitt J, Fabien Soulé V, et al. Biological effect of hydrolyzed collagen on bone metabolism. Crit Rev Food Sci Nutr 2017;57:1922-37. https://doi.org/10.1080/1 0408398.2015.1038377. 
